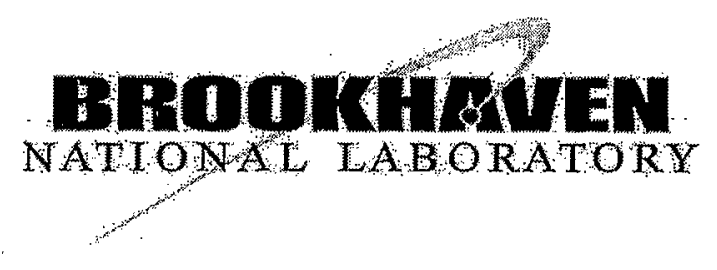

BNL-77354-2006-CP

\title{
Unique Description for Single Transverse Spin Asymmetries in DIS and Hadronic Collisions
}

\author{
Feng Yuan \\ Presented at the $17^{\text {th }}$ International Spin Physics Symposium, Spin 2006 \\ Kyoto, Japan \\ October 2-7, 2006
}

December 2006

\author{
Physics Department/ RIKEN BNL Research Center \\ Brookhaven National Laboratory \\ P.O. Box 5000 \\ Upton, NY 11973-5000 \\ wWw.bnl.gov
}




\section{DISCLAIMER}

This report was prepared as an account of work sponsored by an agency of the United States Government. Neither the United States Government nor any agency thereof, nor any of their employees, nor any of their contractors, subcontractors, or their employees, makes any warranty, express or implied, or assumes any legal liability or responsibility for the accuracy, completeness, or any third party's use or the results of such use of any information, apparatus, product, or process disclosed, or represents that its use would not infringe privately owned rights. Reference herein to any specific commercial product, process, or service by trade name, trademark, manufacturer, or otherwise, does not necessarily constitute or imply its endorsement, recommendation, or favoring by the United States Government or any agency thereof or its contractors or subcontractors. The views and opinions of authors expressed herein do not necessarily state or reflect those of the United States Government or any agency thereof. 


\title{
Unique Description for Single Transverse Spin Asymmetries in DIS and Hadronic Collisions
}

\author{
Feng Yuan
}

\author{
RIKEN/BNL Research Center, Building 510A, Brookhaven National Laboratory, Upton, NY $11973^{1}$
}

\begin{abstract}
We derive a unique formula for the single-transverse-spin asymmetry in semi-inclusive hadron production in deep inelastic scattering, valid for all transverse momentum region. Based on this, we further study the integrated asymmetry weighted with transverse-momentum. They can be evaluated in terms of the twist-three quark-gluon correlation functions, which are responsible for the single spin asymmetry in single inclusive hadron production in hadronic collisions. By using the fitted twist-three functions from the hadronic collision data, we find a consistent description for SSAs in deep inelastic scattering. This demonstrates that we have a unique picture for SSAs in these two processes, and shall provide important guidelines for future studies.
\end{abstract}

Keywords: Single transverse-spin asymmetries

PACS: $12.38 . \mathrm{Bx}, 12.39 . \mathrm{St}, 13.85 . \mathrm{Qk}$

Single-transverse spin asymmetry (SSA) is a novel phenomena in hadronic reactions [1], and has long been observed in various processes $[2,3]$. Recent experimental activities have motivated much theoretical developments for understanding the underlying physics associated with SSA phenomena. Two mechanisms have been proposed in Quantum Chromodynamics (QCD) to explain the large size of SSAs. One follows the collinear (CO) QCD factorization approach, and presents the SSAs in terms of spindependent twist-three quark-gluon correlation functions $[4,5]$. The other explicitly connects the SSAs to spin dependence of partons' transverse motion in a polarized proton, and expresses the SSAs in terms of naive time-reversal-odd (T-odd) and transversemomentum dependent (TMD) parton distributions [6, 7].

In our recent publications [8], we have shown that these two mechanisms are unified for the SSA in the semi-inclusive hadron production in Deep Inelastic Scattering (SIDIS) and Drell-Yan lepton pair production in hadronic collisions. For example, in SIDIS, at large $P_{h \perp} \sim Q$, the quark-gluon correlation approach applies. At small $P_{h \perp} \ll Q$, a factorization in terms of TMD parton distribution applies [9], involving in case of the SSA the Sivers functions. If $P_{h \perp}$ is much larger than $\Lambda_{\mathrm{QCD}}$, the dependence of these functions on transverse momentum may be computed using QCD perturbation theory. At the same time, the result obtained within the twist-three formalism may also be extrapolated into the regime $\Lambda_{\mathrm{QCD}} \ll P_{h \perp} \ll Q$, and we demonstrated that the result of this extrapolation is identical to that obtained using the TMD approach [8]. In this sense, we have unified the two mechanisms widely held responsible for the observed

\footnotetext{
1 We are grateful to RIKEN, Brookhaven National Laboratory and the U.S. Department of Energy (contract number DE-AC02-98CH10886) for providing the facilities essential for the completion of their work.
} 
SSAs.

The differential cross section for SIDIS at $P_{h \perp} \ll Q$ can be written as,

$$
\frac{d \sigma^{\mathrm{TMD}}\left(S_{\perp}\right)}{d x_{B} d y d z_{h} d^{2} \vec{P}_{h \perp}}=\sigma_{0} \times\left[F_{U U}-\sin \left(\phi_{h}-\phi_{S}\right)\left|S_{\perp}\right| F_{U T}^{\text {sivers }}\right]
$$

where $\sigma_{0}=4 \pi \alpha_{\mathrm{em}}^{2} S_{e p} / Q^{4} \times\left(1-y+y^{2} / 2\right) x_{B}$, and $\phi_{S}$ and $\phi_{h}$ are the azimuthal angles of the proton's transverse polarization vector and the transverse momentum vector of the final-state hadron, respectively. Here the azimuthal angles are defined in the so-call virtual photon frame where the virtual photon is moving in the $+z$ direction. $F_{U U}$ and $F_{U T}^{\text {sivers }}$ depend on the kinematical variables, $x_{B}, z_{h}, Q^{2}, y$, and $P_{h \perp}$. According to the TMD factorization formalism, these structure functions can be factorized into products of TMD parton distributions and fragmentation functions, and soft and hard parts: For example, $F_{U T}^{\text {sivers }}$ has the following factorized form [9]:

$$
\begin{aligned}
F_{U T}^{\text {sivers }}= & \sum_{q=u, d, s, \ldots} e_{q}^{2} \int d^{2} \vec{k}_{\perp} d^{2} \vec{p}_{\perp} d^{2} \vec{\lambda}_{\perp} \frac{\vec{k}_{\perp} \cdot \hat{\vec{p}}_{h \perp}}{M_{P}} q_{T}\left(x_{B}, k_{\perp}\right) \\
& \times \hat{q}\left(z_{h}, p_{\perp}\right)\left(S\left(\vec{\lambda}_{\perp}\right)\right)^{-1} H_{U T}^{(1)}\left(Q^{2}\right) \delta^{(2)}\left(z_{h} \vec{k}_{\perp}+\vec{p}_{\perp}+\vec{\lambda}_{\perp}-\vec{P}_{h \perp}\right)
\end{aligned}
$$

where $\hat{\vec{P}}_{h \perp}$ is a unit vector in direction of $\vec{P}_{h \perp}$, and $\hat{q}$ and $q_{T}$ are unpolarized quark fragmentation function and the Sivers TMD quark distribution, respectively.

At large transverse momentum $P_{h \perp} \gg \Lambda_{\mathrm{QCD}}$, we can calculate the same quantity using the twist-three quark-gluon correlation functions [8]. Based on the results in [8], we can write down a unique formula for the transverse momentum dependence. Following the procedure of [10], the differential cross section for the spin dependent SIDIS process can be written as,

$$
\frac{d \Delta \sigma\left(S_{\perp}\right)}{d y d x_{B} d z_{h} d^{2} P_{h \perp}}=\frac{d \Delta \sigma^{\mathrm{TMD}}}{d y d x_{B} d z_{h} d^{2} P_{h \perp}}+\left(\frac{d \Delta \sigma^{\mathrm{CO}}}{d y d x_{B} d z_{h} d^{2} P_{h \perp}}-\left.\frac{d \Delta \sigma^{\mathrm{CO}}}{d y d x_{B} d z_{h} d^{2} P_{h \perp}}\right|_{P_{h \perp} \ll Q}\right)
$$

which is valid to all transverse momentum region at leading power of $1 / Q^{2}[10]$. In the above equation, the first term comes from the TMD factorization formalism, and second term from the collinear factorization with the twist-three quark-gluon correlations contributions. The second term will dominate the SSA at large transverse momentum, and its $P_{\perp}$-dependence can be calculated from perturbative QCD. On the other hand, at low transverse momentum $P_{h \perp} \ll Q$, the second term vanishes, because the two contributions are exactly the same in this limit, and cancel out each other. Experimentally, if we can study the transverse momentum dependence of the SSA for a wide range, we shall explore the transition from perturbative region to the nonperturbative region.

Currently, the experimental study in SIDIS has limited access to the large transverse momentum SSA, and most of the data are in the low transverse momentum region, where the TMD formalism dominates. In phenomenological studies, in order to compare with the experimental data, one has to make a model assumption for the $P_{\perp}$-dependence of the distribution and fragmentation functions [11]. However, one can further study 
the transverse momentum weighted single spin asymmetries, where the $p_{\perp}$-integral for various factors in the factorization formula (2) decouple from each other without detailed modelling for the $P_{\perp}$-dependence [12], and the differential cross section can be written as

$$
\int d^{2} P_{h \perp} \frac{2 P_{h \perp}}{M_{P}} \sin \left(\phi_{h}-\phi_{S}\right) \frac{d \Delta \sigma^{\mathrm{TMD}}\left(S_{\perp}\right)}{d x_{B} d y d z_{h} d^{2} \vec{P}_{h \perp}}=\sigma_{0} \frac{z_{h}}{M_{P}} g_{s} T_{F}\left(x_{B}\right) \hat{q}\left(z_{h}\right),
$$

where $T_{F}(x)$ is the Qiu-Sterman matrix element of the quark-gluon correlation function, and is defined as [5]

$$
T_{F}(x)=\int \frac{d \xi^{-} d \eta^{-}}{4 \pi} e^{i\left(x P^{+} \eta^{-}\right)} \varepsilon_{\perp}^{\beta \alpha} S_{\perp \beta}\left\langle P S\left|\bar{\psi}(0) \gamma^{+} F_{\alpha}{ }^{+}\left(\xi^{-}\right) \psi\left(\eta^{-}\right)\right| P S\right\rangle,
$$

and it is related to the $k_{\perp}$-moment of the TMD quark Sivers function as [13],

$$
\int d^{2} \vec{k}_{\perp} \frac{\vec{k}_{\perp}^{2}}{M_{P}} q_{T}\left(k_{\perp}, x\right)=-g_{s} T_{F}(x)
$$

where $g_{s}$ is the strong coupling constant. And then, the $P_{\perp}$-weighted SSA can be calculated,

$$
\frac{\left\langle 2 \frac{P_{h \perp}}{M_{P}} \sin \left(\phi_{h}-\phi_{S}\right)\right\rangle_{\mathrm{UT}}}{\langle 1\rangle_{\mathrm{UU}}}=\frac{\int \frac{1}{Q^{4}}\left(1-y+\frac{y^{2}}{2}\right) x_{B} \frac{z_{h}}{M_{P}} \sum_{q} e_{q}^{2} g_{s} T_{F}^{q}\left(x_{B}\right) \hat{q}\left(z_{h}\right)}{\int \frac{1}{Q^{4}}\left(1-y+\frac{y^{2}}{2}\right) x_{B} \Sigma_{q} e_{q}^{2} q\left(x_{B}\right) \hat{q}\left(z_{h}\right)} .
$$

The quark-gluon correlation functions $T_{F}^{q}$ have recently been fit to the single inclusive hadron SSA in hadronic collisions [1.4], with the following parameterizations,

$$
T_{F}^{a}(x)=N_{a} x^{\alpha_{a}}(1-x)^{\beta_{a}} q_{a}(x),
$$

where $q_{a}(x)$ is the unpolarized quark distribution for flavor $a$. Two sets of fit were obtained: one with two-flavor fit for u- and d-quark only; other one for both the valence and sea flavors. For details, please refer to [14]. In Fig. 1, we compare our predictions for the $P_{\perp}$-weighted SSA in SIDIS from Eq. (7) with the fitted $T_{F}^{q}$ from [14] to the preliminary experimental data from HERMES collaboration [15]. From these comparisons, we find both fits' predictions roughly agree with the experimental data ${ }^{2}$, especially for the signs.

This is a very nontrivial comparison, because the SSA in DIS comes from the final state interaction, whereas in hadronic reactions both initial and final state interactions contribute and they weight with different hard factors especially the color factors [14]. All these effects have to be taken into account to achieve the above comparison. In other words, it is the nontrivial physics (induced by the initial/final state interactions) that leads to the consistent description for the single spin asymmetries in both processes. This demonstrates that we indeed have a unique picture for single transverse spin asymmetries in DIS and hadronic collisions.

We thank collaborations and discussions with Xiangdong Ji, Jianwei Qiu, and Werner Vogelsang.

\footnotetext{
2 These data have also been fitted to the quark Sivers functions in [16].
} 


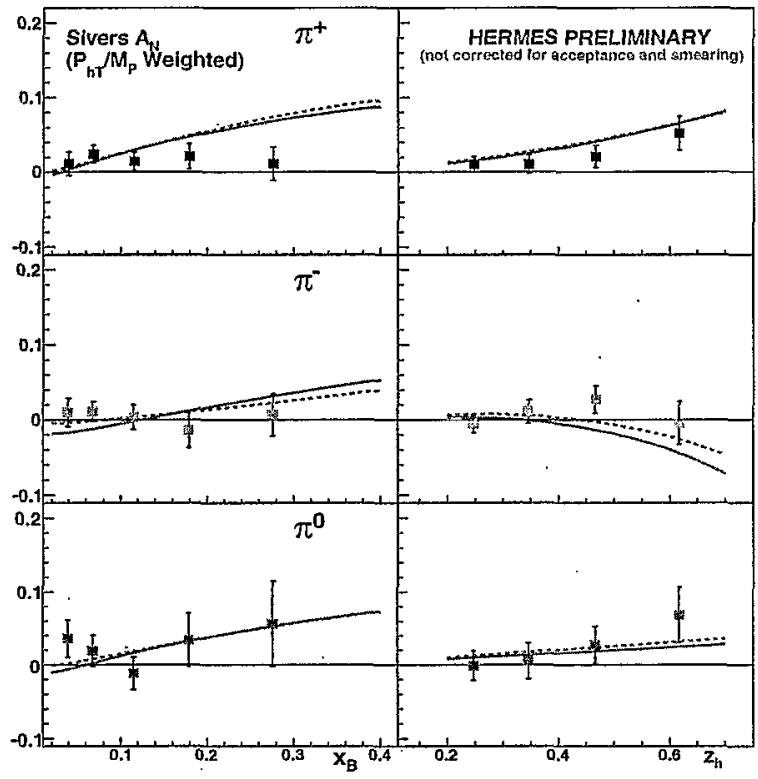

FIGURE 1. $P_{h \perp}$-weighted SSAs in SIDIS calculated from the quark-gluon correlation functions $T_{F}^{q}$, which were fitted to SSA data in hadronic experiments. The predictions are compared to the preliminary data from HERMES [15]. The solid line represents the results from the Fit I parameterizations for $T_{F}$, and the dashed line for Fit II [14].

\section{REFERENCES}

1. M. Anselmino, A. Efremov and E. Leader, Phys. Rept. 261, 1 (1995) [Erratum-ibid. 281, 399 (1997)]; Z. t. Liang and C. Boros, Int. J. Mod. Phys. A 15, 927 (2000); V. Barone, A. Drago and P. G. Ratcliffe, Phys. Rept. 359, 1 (2002).

2. G. Bunce et al., Phys. Rev. Lett. 36, 1113 (1976).

3. D. L. Adams et al. , Phys. Lett. B 261, 201 (1991); D. L. Adams et al., Phys. Lett. B 264, 462 (1991); K. Krueger et al., Phys. Lett. B 459, 412 (1999).

4. A. V. Efremov and O. V. Teryaev, Șov. J. Nucl. Phys. 36, 140 (1982) [Yad. Fiz. 36, 242 (1982)]; Phys. Lett. B 150, 383 (1985).

5. J.W. Qiu and G. Sterman, Phys. Rev. Lett. 67, 2264 (1991); Nucl. Phys. B 378, 52 (1992); Phys. Rev. D 59, 014004 (1999).

6. D. W. Sivers, Phys. Rev. D 41, 83 (1990); Phys. Rev. D 43, 261 (1991).

7. S. J. Brodsky, D. S. Hwang and I. Schmidt, Phys. Lett. B 530, 99 (2002); Nucl. Phys. B 642, 344 (2002); J. C. Collins, Phys. Lett. B 536, 43 (2002); X. Ji and F. Yuan, Phys. Lett. B 543, 66 (2002); A. V. Belitsky, X. Ji and F. Yuan, Nucl. Phys. B 656, 165 (2003).

8. X. Ji, J. W. Qiu, W. Vogelsang and F. Yuan, Phys. Rev. Lett. 97, 082002 (2006); Phys. Rev. D 73, 094017 (2006); Phys. Lett. B 638, 178 (2006).

9. X. Ji, J. P. Ma and F. Yuan, Phys. Rev. D 71, 034005 (2005); Phys. Lett. B 597, 299 (2004); J. C. Collins and A. Metz, Phys. Rev. Lett. 93, 252001 (2004).

10. J. C. Collins, D. E. Soper and G. Sterman, Nucl. Phys. B 250, 199 (1985).

11. see for example, M. Anselmino et al., arXiv:hep-ph/0511017.

12. D. Boer and P. J. Mulders, Phys. Rev. D 57, 5780 (1998).

13. D. Boer, P. J. Mulders and F. Pijlman, Nucl. Phys. B 667, 201 (2003).

14. C. Kouvaris, J. W. Qiu, W. Vogelsang and F. Yuan, arXiv:hep-ph/0609238.

15. See talks by N. Makins at QCD'N 2004 Workshop at Trento, and by R. Seidl, at the DIS 2004 Workshop at Madision, Wisconsin.

16. A. V. Efremov, K. Goeke, S. Menzel, A. Metz and P. Schweitzer, Phys. Lett. B 612, 233 (2005). 